\title{
The ratio of negative lymph nodes count inside the thoracic cavity to the positive lymph count is a prognostic factor in esophageal squamous cell carcinoma
}

\author{
jinling Zhang \\ Linyi People's Hospital \\ yan Liu \\ Linyi People's Hospital \\ hongyan Li \\ Linyi People's Hospital \\ xinglan $\mathrm{Li}$ \\ Linyi People's Hospital \\ luning $\mathrm{Li}$ \\ Linyi People's Hospital \\ lianling Yu \\ Linyi People's Hospital \\ fengyuan Che \\ Linyi People's Hospital \\ xueyuan Heng ( $\nabla$ xueyuanheng@yahoo.com ) \\ Linyi People's Hospital https://orcid.org/0000-0002-8174-0347
}

\section{Research}

Keywords: esophageal cancer, tumor metastasis, lymph node number, thoracic cavity

Posted Date: October 29th, 2020

DOI: https://doi.org/10.21203/rs.3.rs-96696/v1

License: (c) (1) This work is licensed under a Creative Commons Attribution 4.0 International License. Read Full License

Version of Record: A version of this preprint was published at Journal of Clinical Oncology on May 20th, 2019. See the published version at https://doi.org/10.1200/JC0.2019.37.15_suppl.e15545. 


\section{Abstract}

BACKROUND: It was reported that the number of positive lymph nodes (PLN) and negative lymph nodes (NLN) resected in lymphadenectomy were both independent prognostic factors in esophageal squamous cell carcinoma (ESCC). However, it is unclear which kind of combination of them would have a stronger impact on the prognosis of the ESCC patients.

METHODS: A total of 229 patients with ESCC were retrospectively investigated. The Cox Proportional Hazards Model was used to investigate the relationship between the PLN or NLN and the overall survival of patients. Survival analysis was performed using the Kaplan-Meier method, and the survival difference between sub-groups were explored with log-rank tests. The cut-off number of the ratio of NLN count to PLN count resulted in an improved outcome for patients was investigated by a series of log-rank tests.

RESULTSs: The numbers of PLN and NLN were both independent prognostic factors, supported by the results of Cox regression model analysis (hazard ratio $[H R], 1.12, P<0.001$ and $H R, 0.97, P=0.024$, respectively). The survival differences among sub-groups from N0 to N3 stage were statistically significant $(P<0.001)$. Analysis using a Cox regression model revealed that the number of NLN inside the thoracic cavity had a stronger impact on the prognosis compared to the number of NLN outside the thoracic cavity $(H R, 0.959, P=0.042$ vs. $H R, 0.973, P=0.179)$. As a combination analysis consisting of the number of PLN and NLN, the log-rank test result demonstrated that patients with the ratio of NLN count inside thoracic cavity to the PLN count less than 8 could achieve a better survival.

CONCLUSIONS: The ratio of NLN count inside thoracic cavity to the PLN count was a independent prognostic factor.

\section{Introduction}

Esophageal squamous cell carcinoma (ESCC) is the major pathological type of esophageal cancer in east Asia, where as adenocarcinoma is the main pathological type in north America and Europe(1). Lymph node metastasis (LNM), or positive lymph nodes (PLN) usually occurs early in the development of ESCC, which have a prominent impact on the evaluation of tumor stage and the prognosis of patients(24).

It is widely accepted that the number of PLN removed in the lymphadenectomy is closely associated with the prognosis of patients with $\operatorname{ESCC}(5,6)$.Moreover, the $\mathrm{N}$ stage of the 7th edition of TNM tumor staging system from the American Joint Committee on Cancer (AJCC)was defined using the number of PLN in ESCC. However, recently, several researchers have reported that the number of negative lymph nodes $(\mathrm{NLN})$ is also a significant prognostic factor in patients with $\operatorname{ESCC}(7)$.

The number of NLN that should be removed via lymphadenectomy in patients with ESCC remains a controversial topic. Hsu reported that ESCC patients would benefit from the lymphadenectomy if more than 18 NLN were removed during surgery. In other words, this research suggested that 
lymphadenectomy in ESCC was only appropriate if at least19 NLN were identified(7). Furthermore, another researcher proposed that lymphadenectomy in which more than $21 \mathrm{NLN}$ are removed is a more appropriate method(8).

It has also been reported that the site of PLN and NLN that are removed during lymphadenectomy may be a more important predictor of prognosis than the number of $\operatorname{PLN}$ and $\operatorname{NLN}(9,10)$. In our previous study, we also confirmed that certain sites of NLN may play important roles in the prognosis of patients with $\operatorname{ESCC}(11)$.

Since the site and number of PLN or NLN are both possible predictors of outcome in patients with ESCC, it is necessary to investigate their effects on the prognosis of ESCC. It is widely accepted that patients with tumor metastasis occurring outside the thoracic cavity in ESCC will have poorer outcomes compared with other ESCC patients(12-14). It is more common for NLN to be identified inside the abdominal cavity rather than the thoracic cavity in healthy people. Consequently, it is necessary to compare the influence of the number of NLN inside and outside the thoracic cavity on the prognosis in ESCC. This study aimed to explore the impact of NLN inside the thoracic cavity on the prognosis of patients, compared to NLN outside the thoracic cavity, and further investigated the combination impact of the PLN and NLN on prognosis of ESCC patients.

\section{Material And Methods}

\section{Patient selection}

229 ESCC patients who experienced esophagectomy and lymphadenectomy between Jan 2009 and Jan 2013 were retrospectively followed in this research. This study was approved by the Institutional Review Board of Linyi People's Hospital affiliated hospital to Shandong University.

The inclusion criteria was described as follows: all patients received upper gastrointestinal barium radiography and upper gastrointestinal endoscopy, and the site of primary tumor was confirmed in the thoracic esophagus; ultrasound of the cervical region, computed tomography of chest and upper abdomen were performed to explore distant metastasis; only the patient with Karmofsky Performance Status score more than 70 were considered; neo-adjuvant radiotherapy and/or neo-chemotherapy were not delivered on patients; the primary tumor was identified as ESCC by pathologist after surgery; tumorfree resection margins were confirmed.

\section{Treatment}

All patients had undergone extended esophagectomy with three-field or two-field lymph node dissection. The patients were received three-field lymph node dissection when tumor metastasis in the cervical region was considered based on the result of ultrasound or computed tomography examination. All the threefield lymph node dissection consisted of a collar neck incision, a right-sided thoracotomy and an upper 
midline laparotomy comparing to the two-field lymph node dissection which only included a right-sided thoracotomy and a upper midline laparotomy.

The lymph nodes dissected during operation were labeled using the lymph node station nomination of the Japanese Society for Esophageal Diseases (JSED). The removed specimens including the lymph nodes and the primary tumor were collected at the end of operation by surgeons, and were further evaluated by experienced pathologists to count the number of PLN and NLN in every lymph node station after the primary tumor was identified as ESCC.

The entire specimens were fixed in the $10 \%$ formalin immediately after operation, and then they were embedded in paraffin. After 24 hours, these specimens were received hematoxylin and eosin staining after being cut into $5-\mu \mathrm{m}$ slices. The PLN and NLN were evaluated by two pathologists and the number of PLN and NLN in each lymph node station were recorded after the two pathologists discussed and reached an agreement.

The pathological tumor stage of each case was evaluated and recorded according to TNM system of AJCC (the 7th edition).

Postoperative radiochemotherapy was delivered to the ESCC patient with high-risk factors which were identified by pathologist after operation. Patients with high-risk factors were administrated with 5fluorouracil-based concurrent radiotherapy. The total dose of radiation therapy was in a interval of $54 \sim$ $60 \mathrm{~Gy}$.

\section{Follow-up}

All patients were evaluated every 3 months for the first two years, every 6 months during the next three years, and annually thereafter. The follow-up examinations on patients were as follows: history taking, physical examination, upper gastrointestinal barium radiography, CT/MR of chest and upper abdomen. The emphases of follow-up examinations were used to evaluate the local recurrences and remote metastases. Patients who could not come back to our hospital regularly were followed up by phone. The last follow-up time was Jan 2016. Overall survival was calculated from the date of operation. Surviving patients were censored on the date of the last follow-up. We confirmed the survival and the death by telephone or medical record review.

\section{Statistical analysis}

Continuous variates were summarized by medians and ranges. The relationships between clinicopathological factors and prognosis were explored using forward stepwise Cox proportional hazard regression model analysis. Survival analysis was performed using Kaplan-Meier method and the statistical difference of overall survival between the subgroup of patients was investigated by log-rank test. The influential cut-off numbers of PLN or NLN on prognosis of patients were investigated using the comparison among the result of a series of log-rank tests. 


\section{Results}

\section{Patients and clinicopathological features}

A total of 229 ESCC patients were included in this study. The median age was 63 years (range, 30-79 years). The patients consisted of 208 males and 21 females.Tumors were located in the upper esophagus in 9 patients, in the middle esophagus in 128 patients and in the lower esophagus in 82patients. The tumor site in the last 10 patients were difficult to be identified into the above three site, because they all were located in two sites of them in the meantime. In terms of macroscopic types, there were 146 patients with exophytic type and 83 patients with endophytic type. The median tumor length was $4.5 \mathrm{~cm}$ (range, $1.2-13 \mathrm{~cm}$ ). Other clinical and pathological characteristics of patients in this study are presented in Table 1 , including tumor macroscopic type, tumor differentiation, T stage, nerve plexus invasion, the results of immunohistochemical analysis and adjuvant treatment schemes.

Tumor metastasis in regional lymph nodes was identified in 109 of 229 cases.

\section{Outcome of follow-up}

The median time of follow-up was 22.5 months (range, 3-73 months). Up to January 2016, follow-up was unsuccessful in 9 cases due to wrong phone numbers and the follow-up rate was $96.1 \%$. The 1-, 3- and 5year survival rates in this study were $72.0,47.7$ and $20.5 \%$,respectively.

\section{Factors associated with overall survival}

Results of univariate and multivariate analysis with the Cox proportional hazard regression model are presented in Table 2. Tumor differentiation, the number of PLN, and the ratio of PLN to total lymph nodes were significant predictors for overall survival in the survival analysis. Nevertheless, only tumor differentiation, the number of PLN and the number of NLN were independent prognostic factors for the outcome of patients in the multivariate analysis.

\section{Comparison of overall survival between patients according to different $\mathrm{T}$ stages, tumor differentiation and $\mathrm{N}$ stages}

Patients in this study were classified into four subgroups according to the T stage of the tumor. Nevertheless, statistical differences were not observed among the four subgroups by log-rank test in survival analyses (Fig. 1A). Patients were classified into three subgroups according to tumor differentiation. Significant differences in the overall survival among groups were identified using the logrank test (Fig. 1B). The survival difference was also statistically significant among four subgroups of patients with different N stages, which were N0 (NLN), N1 (1-5 PLN), N2 (6-9 PLN) andN3 ( 110 PLN) (Fig. 1C).

The effect of NLN inside the thoracic cavity on the prognosis of ESCC 
Beside the number of NLN, this study further explored whether the site of NLN had a significant impact on the prognosis of patients with ESCC. Similar to previous findings in ESCC, tumor differentiation and the number of PLN were both confirmed as independent prognostic factors in this study. However, the results of this study also revealed that the number of NLN inside the thoracic cavity, instead of the number of NLN outside the thoracic cavity, was an independent prognostic factor in the Cox proportional hazard regression model. Table 3 presented the detailed results of univariate and multivariate analyses for overall survival by Cox proportional hazard regression model.

\section{The cut-off number of the ratio of NLN count to PLN count on survival}

In the investigation on the combined impact of PLN and NLN on prognosis of patients, this study tried to find a cut-off number in below the three kind of ratio. The first was the ratio of the total NLN count to the PLN count (Ratio ${ }^{N L N t o t a l / P L N}$ ), the second was the ratio of the NLN count inside thoracic cavity to the PLN count (Ratio ${ }^{\text {NLNinside/PLN }}$ ) and the third was the ratio of the NLN outside thoracic cavity to the PLN count (Ratio ${ }^{\text {NLNoutside/PLN }}$ ). Only the cut-off number of Ratio ${ }^{\text {NLNinside/PLN }}$ was identified, which patients with the ratio less than 8 would have a better survival. In another word, more NLN inside thoracic cavity removed in the lymphadenectomy could not bring a better survival for patients. The numeric distribution of RatioNLNinside/PLN was displayed using a dot plot (Fig 2A). The cut-off number of Ratio ${ }^{\text {NLNinside/PLN }}$ dividing patients into group $A$ and group $B$, and the result of log-rank test revealed that patients with the Ratio ${ }^{\text {LNNinside/PLN }}$ more than 8 (including 8) could lead to the worse outcome of patients comparing to others (Fig 2 B to Fig 2F).

\section{Discussion}

The status of LNM in ESCC is essential for evaluating the tumor stage and selection of post-operative treatment $(15,16)$. The classification on tumor stage of AJCC (7th edition) is primarily based on the number of PLN rather than the number of NLN, since the majority of previous studies have demonstrated that the number of PLN is an independent prognostic factor in ESCC and an indicator for the extent of lymphadenectomy(17).

However, recent reports have shown that the number of NLN also plays an important role in the prognosis of patients with ESCC that have undergone radical resection of $\operatorname{ESCC}(18,19)$. The current study also confirmed that the number of NLN removed in lymphadenectomy was closely associated with the outcome of patients, and further investigation revealed the number of NLN inside the thoracic cavity was an independent predictor for prognosis while the number of NLN outside the thoracic cavity was not an independent prognostic factor. This finding was partly consistent with several studies, which reported that the site of PLN is a more important prognostic factor in ESCC than the number of PLN $(12,20,21)$.

Based on the results of the this study, it may not be appropriate to evaluate the prognosis of the ESCC patients only based on the number of PLN. This study identified the Ratio ${ }^{N L N i n s i d e / P L N}$ not the Ratio $^{\text {NLNoutside/PLN }}$ or Ratio ${ }^{\text {NLNtotal/PLN }}$ could be used as an indicator for prognosis in ESCC, which implied 
that the number of NLN inside thoracic cavity was also an important factor in prognosis. The reason for this is that the number of NLN outside the thoracic cavity could be misleading, since it may include the normal and physiological swollen lymph nodes which usually occur in the abdominal cavity. Furthermore, the number of normal but physiological swollen lymph nodes inside the abdominal cavity is usually greater than that inside the thoracic cavity, which lead to the collection by mistake in lymphadenectomy.

According to the results of our previous research and other studies, lymph node sites around the esophagus had a higher probability occurring tumor metastasis in thoracic ESCC than lymph node stations outside the thoracic cavity, regardless of the location of the primary tumor(22-24). This indicated that the thoracic cavity plays an important role in the tumor metastasis in thoracic ESCC. One reason of this may be that the lymphatic network around the esophagus is the first site of tumor metastasis in ESCC, leading to a high probability of tumor metastasis. Another reason may be that the diaphragm muscle has a blocking effect on tumor metastasis via lymphatic vessels.

This study demonstrated that the combination of the number of NLN inside thoracic cavity and the number of PLN was a strong prognostic factor. Furthermore, on basis of the result of this study, the extent of lymphadenectomy with NLN count inside thoracic cavity 8 times higher of than PLN count were not persuaded to proceed. This finding was consistent to the result of two recent published research, which emphasized that redundant LN removed in lymphadenectomy could not bring benefit for patients' survival (25-26).

Although researchers have tried to set up a modified $\mathrm{N}$ stage referring to the principle of $\mathrm{N}$ stage in stomach cancer which defined the $\mathrm{N}$ stage according to the site of PLN, few reliable results have been obtained until now(27). The reason for this might be that the anatomical structure and site of the esophagus and stomach are different, which leaded to different features of LNM between esophageal cancer and stomach cancer. It may be inappropriate to only use the site of PLN to evaluate the N stage in ESCC. A more reasonable approach would be to incorporate the site and number of lymph nodes consisting of PLN and NLN to evaluate the $\mathrm{N}$ stage in ESCC in the future.

At present, it is not clear which site of PLN had a more important effect on prognosis than others. The reason for this may be that it is more reliable to use the number of NLN rather than PLN to evaluate the extent of lymphadenectomy in every regional lymph node site in ESCC, which was supported by a research focusing on the comparison on the impact effect in prognosis between the site and the number of PLN(28). Using NLN to evaluate N stage in ESCC was a novel method, but no stable result on the modified N stage only using the number of NLN were reported. The reason might be that the site of NLN should be considered in the modified $\mathrm{N}$ stage based on the findings mentioned above. The Ratio $^{\text {NLNinside/PLN }}$ might be useful parameter in the promotion of the modified $\mathrm{N}$ stage in future.

The present study demonstrated that the number of PLN and tumor differentiation were independent prognostic factors, which was consistent with previous results(29). This finding indicated that this study had a low risk of sample selection bias. 
The present study revealed that the hazard ratio of tumor differentiation for prognosis was higher than that for tumor T stage or tumor length, indicating that tumor differentiation was more sensitive and stable in the Cox proportional hazard model than tumor length or tumor T stage. The reason for this result might be that the accuracy rate on the tumor length and tumor T stage would be influenced by the detection site of pathology. This finding was consistent with the results of our previous research, which proposed a difference between poorly differentiated ESCC and middle or well differentiated ESCC.

In summary, this study demonstrated that the number of NLN inside the thoracic cavity maybe a stronger indicator for the extent of lymphadenectomy and prognosis in ESCC than the number of NLN outside the thoracic cavity. Furthermore, this study proposed that the Ratio ${ }^{\text {NLNinside/PLN }}$ basing on the combination of the PLN and NLN was a useful prognostic factor and identified a cut-off number of it, which indicated the appropriate extent of lymphadenectomy for the ESCC patients.

Although this study presented two novel findings, as mentioned above, there were several limitations that should be mentioned. Firstly, since the limited sample size of this study could lead to statistical bias, the research had replicated the result under the strict statistical method avoiding obvious statistical bias.

Secondly, this study failed to promote a modified $\mathrm{N}$ stage based on the finding of Ratio ${ }^{\text {NLNinside/PLN }}$. The reason might have relation with the limit cohort in this study. However, another large sample of prospective investigation has been prepared, for which a novel method on $\mathrm{N}$ stage classification system would be achieved in the near future.

\section{Conclusions}

The ratio of NLN count inside thoracic cavity to the PLN count was a independent prognostic factor. The ratio have the hope not only to be used as an indicator for the extent of lymphadenectomy but also to be used as a parameter to evaluate the quality of the lymphadenectomy for the selection of post-operation treatment.

\section{Abbreviations}

\section{PLN}

positive lymph nodes

NLN

negative lymph nodes

ESCC

esophageal squamous cell carcinoma

HR

hazard ratio

LNM

Lymph node metastasis

AJCC 


\section{Declarations}

\section{Acknowledgments}

Not applicable.

\section{Funding}

The present study was supported by Shandong Provincial Medical and Health Development Plan (grant no. 2013WSA13018, Dr. Jinling Zhang), the Natural Science Foundation of Shandong Province (grant no. ZR2013HL018 and ZR2014HL062, Dr. Jinling Zhang).

\section{Availability of data and materials}

The datasets used and/or analyzed during the present study are available from the corresponding author on reasonable request.

\section{Authors' contributions}

$\mathrm{XH}$ and $\mathrm{FC}$ designed the study. $\mathrm{XL}$ and $\mathrm{LL}$ collected the information of patients and analyzed the data. JZ and $\mathrm{YW}$ wrote the paper. $\mathrm{HZ}, \mathrm{YL}$ and $\mathrm{LY}$ reviewed and edited the manuscript. All authors read and approved the manuscript.

All procedures used in the present study involving human participants were in accordance with the ethical standards of the institutional research committee, and with the 1964 Helsinki declaration and its later amendment or comparable ethical standards. The present study was approved by the Ethics Review Board of Linyi People's Hospital (Shangdong, China) and written informed consent was collected from each patient.

\section{Consent for publication}

Informed consent was obtained from all participants included in the study.

\section{Competing interests}

The authors declare that they have no competing interests.

\section{References}

1. Gockel I, Niebisch S, Ahlbrand CJ, et al.: Risk and Complication Management in Esophageal Cancer Surgery: A Review of the Literature. The Thoracic and cardiovascular surgeon 64: 596-605, 2016. 
2. Wang H, Deng F, Liu Q and Ma Y: Prognostic significance of lymph node metastasis in esophageal squamous cell carcinoma. Pathology, research and practice 213: 842-847, 2017.

3. Xu XL, Zheng WH, Zhu SM, Zhao A and Mao WM: The Prognostic Impact of Lymph Node Involvement in Large Scale Operable Node-Positive Esophageal Squamous Cell Carcinoma Patients: A 10-Year Experience. PloS one 10: e0133076, 2015.

4. Wu SG, Li FY, Zhou J, et al.: Prognostic value of different lymph node staging methods in esophageal squamous cell carcinoma after esophagectomy. The Annals of thoracic surgery 99: 284-290, 2015.

5. Zhang D, Zheng Y, Wang Z, et al.: Comparison of the 7th and proposed 8th editions of the AJCC/UICC TNM staging system for esophageal squamous cell carcinoma underwent radical surgery. European journal of surgical oncology : the journal of the European Society of Surgical Oncology and the British Association of Surgical Oncology2017.

6. Yamasaki M, Miyata H, Miyazaki Y, et al.: Evaluation of the nodal status in the 7th edition of the UICC-TNM classification for esophageal squamous cell carcinoma: proposed modifications for improved survival stratification : impact of lymph node metastases on overall survival after esophagectomy. Annals of surgical oncology 21: 2850-2856, 2014.

7. Hsu PK, Huang CS, Wang BY, Wu YC, Chou TY and Hsu WH: The prognostic value of the number of negative lymph nodes in esophageal cancer patients after transthoracic resection. The Annals of thoracic surgery 96: 995-1001, 2013.

8. Baba $\mathrm{Y}$, Watanabe M, Shigaki $\mathrm{H}$, et al.: Negative lymph-node count is associated with survival in patients with resected esophageal squamous cell carcinoma. Surgery 153: 234-241, 2013.

9. Talsma AK, Ong CA, Liu X, et al.: Location of lymph node involvement in patients with esophageal adenocarcinoma predicts survival. World journal of surgery 38: 106-113, 2014.

10. Tanaka $\mathrm{H}$, Ohira $\mathrm{M}, \mathrm{Kubo} \mathrm{N}$, et al.: Association of location of lymph node metastases with postoperative recurrence of esophageal squamous cell carcinoma. Anticancer research 32: 34213426, 2012.

11. Zhang J, Heng X, Luo Y, et al.: Influence of negative lymph node in No 7 on survival of patients with middle thoracic esophageal squamous cell carcinoma. OncoTargets and therapy 9: 1831-1837, 2016.

12. Wang KN, Xu QR and Chen LQ: Impact of lymph-node metastasis site in patients with thoracic esophageal cancer: does the lymph node metastasis site matter? Journal of surgical oncology 104: 334; author reply 333, 2011.

13. Takemura M, Hori T and Fujiwara Y: Clinical outcomes and prognosis after thoracoscopic esophagectomy with two-field lymph node dissection for lower thoracic esophageal cancer. Anticancer research 33: 601-608, 2013.

14. Tanaka $\mathrm{K}$, Yano M, Motoori M, et al.: The significance of abdominal para-aortic lymph node metastasis in patients with lower thoracic esophageal cancer. Diseases of the esophagus : official journal of the International Society for Diseases of the Esophagus 25: 146-152, 2012. 
15. Wang Q, Yu S, Xiao Z, et al.: Residual lymph node status is an independent prognostic factor in esophageal squamous cell Carcinoma with pathologic T0 after preoperative radiotherapy. Radiation oncology 10: 142, 2015.

16. Robb WB, Maillard E and Mariette C: Lymph Node Status After Neoadjuvant Chemoradiotherapy for Esophageal Cancer: Implications for the Extent of Lymphadenectomy. Annals of surgery2015.

17. Huang Y, Guo W, Shi S and He J: Evaluation of the 7(th) edition of the UICC-AJCC tumor, node, metastasis classification for esophageal cancer in a Chinese cohort. Journal of thoracic disease 8: 1672-1680, 2016.

18. Zhu Z, Chen $\mathrm{H}, \mathrm{Yu}$ W, et al.: Number of negative lymph nodes is associated with survival in thoracic esophageal squamous cell carcinoma patients undergoing three-field lymphadenectomy. Annals of surgical oncology 21: 2857-2863, 2014.

19. Ma $\mathrm{M}$, Tang $\mathrm{P}$, Jiang $\mathrm{H}$, et al.: Number of negative lymph nodes as a prognostic factor in esophageal squamous cell carcinoma. Asia-Pacific journal of clinical oncology2016.

20. Kunisaki C, Makino H, Kimura J, et al.: Impact of lymph-node metastasis site in patients with thoracic esophageal cancer. Journal of surgical oncology 101: 36-42, 2010.

21. Sugawara K, Yamashita H, Uemura Y, et al.: Numeric pathologic lymph node classification shows prognostic superiority to topographic $\mathrm{pN}$ classification in esophageal squamous cell carcinoma. Surgery 162: 846-856, 2017.

22. Cheng J, Kong L, Huang W, et al.: Explore the radiotherapeutic clinical target volume delineation for thoracic esophageal squamous cell carcinoma from the pattern of lymphatic metastases. Journal of thoracic oncology : official publication of the International Association for the Study of Lung Cancer 8: 359-365, 2013.

23. Chen J, Pan J, Zheng X, et al.: Number and location of positive nodes, postoperative radiotherapy, and survival after esophagectomy with three-field lymph node dissection for thoracic esophageal squamous cell carcinoma. International journal of radiation oncology, biology, physics 82: 475-482, 2012.

24. Chen X, Chen J, Zheng X, et al.: Prognostic factors in patients with thoracic esophageal carcinoma staged pT1-4aNOMO undergone esophagectomy with three-field lymphadenectomy. Annals of translational medicine 3: 282, 2015.

25. Patti MG: Survival After Lymphadenectomy in Patients With Esophageal Cancer: A Controversial Issue. JAMA surgery 151: 40, 2016.

26. Lagergren J, Mattsson F, Zylstra J, et al.: Extent of Lymphadenectomy and Prognosis After Esophageal Cancer Surgery. JAMA surgery 151: 32-39, 2016.

27. Lin Z, Chen W, Chen Y, et al.: A new classification of lymph node metastases according to the lymph node stations for predicting prognosis in surgical patients with esophageal squamous cell carcinoma. Oncotarget 7: 76261-76273, 2016.

28. Kunisaki C, Makino H, Kimura J, et al.: Impact of lymph-node metastasis site in patients with thoracic esophageal cancer. Journal of surgical oncology 101: 36-42, 2010. 
29. Schiefer Al, Schoppmann SF and Birner P: Lymphovascular invasion of tumor cells in lymph node metastases has a negative impact on survival in esophageal cancer. Surgery 160: 331-340, 2016.

\section{Tables}




\begin{tabular}{|ll|}
\hline Table.1 Clinicopathological features & \\
\hline Parameter & Cases \\
\hline sex & \\
\hline male & 208 \\
\hline female & 21 \\
\hline age(years) & $63(30-79)$ \\
\hline location of tumor & \\
\hline upper & 9 \\
\hline middle & 128 \\
\hline lower & 82 \\
\hline 4 & 18 \\
\hline macroscopic type & \\
\hline 1 & 146 \\
\hline 2 & 67 \\
\hline Tumor length(cm) & $4.5(1.2-13)$ \\
\hline Tumor differentiation & \\
\hline well differention & 114 \\
\hline moderately differention & 58 \\
\hline poorly differention & 123 \\
\hline T stage & 43 \\
\hline T1 & \\
\hline T2 & \\
\hline T3 & \\
\hline T4 & \\
\hline N stage & \\
\hline N0 & \\
\hline N1 & \\
\hline N2 & \\
\hline
\end{tabular}

Page 13/18 


\begin{tabular}{|ll|}
\hline lymph vascular invasion & \\
\hline negative & 217 \\
\hline positive & 12 \\
\hline invasion of nerve & 220 \\
\hline negative & 9 \\
\hline positive & $40(10-80)$ \\
\hline ki-67(\%) & \\
\hline EGFR & 63 \\
\hline negative & 125 \\
\hline positive & \\
\hline p53 & 62 \\
\hline negative & 48 \\
\hline low expression & 46 \\
\hline moderate expression & 34 \\
\hline high expression & 111 \\
\hline adjuvant therapy & 106 \\
\hline none & \\
\hline chemoradiotherapy & \\
\hline
\end{tabular}

Table2 Univariate and multivariate for overall survival Cox proportional hazard regression model 


\begin{tabular}{|lllll|}
\hline Prognostic factors & $\begin{array}{l}\text { P value } \\
\text { (univariate) }\end{array}$ & HR & P value & HR \\
\hline Age(years old) & 0.675 & 1.002 & - & - \\
\hline Sex(male, female) & 0.787 & 0.763 & - & - \\
\hline Tumor location & 0.189 & 1.012 & - & - \\
\hline (upper, middle, lower) & & & & - \\
\hline Tumor length & 0.107 & 1.078 & - & - \\
\hline (continuous) & & & & - \\
\hline Morphology type(1,2) & 0.921 & 0.930 & - & 1.6742 \\
\hline Tumor differentiation & 0.004 & 1.383 & 0.001 & - \\
\hline (well, moderate, poor) & & & & - \\
\hline T stage(T1,T2,T3,T4) & 0.004 & 1.431 & - & - \\
\hline Ki-67(continuous) & 0.439 & 0.190 & 0.057 & - \\
\hline EGFR & 0.044 & 1.135 & - & - \\
\hline p53 & 0.066 & 1.185 & - & -1.9906 \\
\hline total lymph nodes & 0.796 & 1.007 & - & - \\
\hline Negative lymph nodes & 0.107 & 0.986 & 0.024 & - \\
\hline positive lymph nodes & $<0.001$ & 1.137 & $<0.001$ & - \\
\hline rate of lymph nodes & $<0.001$ & 11.405 & - & - \\
\hline
\end{tabular}

Abbreviations: HR, hazard ratio.

Table 3 Further univariate and multivariate for overall survival Cox proportional hazard regression model 


\begin{tabular}{|c|c|c|c|c|}
\hline Prognostic factors & $\begin{array}{l}\text { P value } \\
\text { (univariate) }\end{array}$ & $\mathrm{HR}$ & $\begin{array}{l}\text { P value } \\
\text { (multivariate) }\end{array}$ & $\mathrm{HR}$ \\
\hline Age(years old) & 0.675 & 1.002 & - & - \\
\hline Sex(male, female) & 0.787 & 0.763 & - & - \\
\hline $\begin{array}{l}\text { Tumor location } \\
\text { (upper, middle, lower) }\end{array}$ & 0.189 & 1.012 & - & - \\
\hline $\begin{array}{l}\text { Tumor length } \\
\text { (continuous) }\end{array}$ & 0.107 & 1.078 & - & - \\
\hline Morphology type $(1,2)$ & 0.921 & 0.930 & - & - \\
\hline $\begin{array}{l}\text { Tumor differentiation } \\
\text { (well, moderate, poor) }\end{array}$ & 0.004 & 1.383 & 0.001 & 1.6742 \\
\hline T stage $(\mathrm{T} 1, \mathrm{~T} 2, \mathrm{~T} 3, \mathrm{~T} 4)$ & 0.004 & 1.431 & - & - \\
\hline Ki-67(continuous) & 0.439 & 0.190 & 0.057 & 0.9906 \\
\hline EGFR & 0.044 & 1.135 & - & - \\
\hline p53 & 0.066 & 1.185 & - & - \\
\hline total lymph nodes & 0.796 & 1.007 & - & - \\
\hline positive lymph nodes & $<0.001$ & 1.137 & $<0.001$ & 1.1219 \\
\hline rate of lymph nodes & $<0.001$ & 11.405 & - & - \\
\hline $\begin{array}{l}\text { Negative lymph nodes } \\
\text { within the thoracic cavity }\end{array}$ & 0.008 & 0.972 & 0.042 & 0.9595 \\
\hline $\begin{array}{l}\text { Negative lymph nodes without the thoracic } \\
\text { cavity }\end{array}$ & 0.681 & 0.999 & 0.179 & 0.9727 \\
\hline
\end{tabular}

Abbreviations: HR, hazard ratio.

\section{Figures}


A

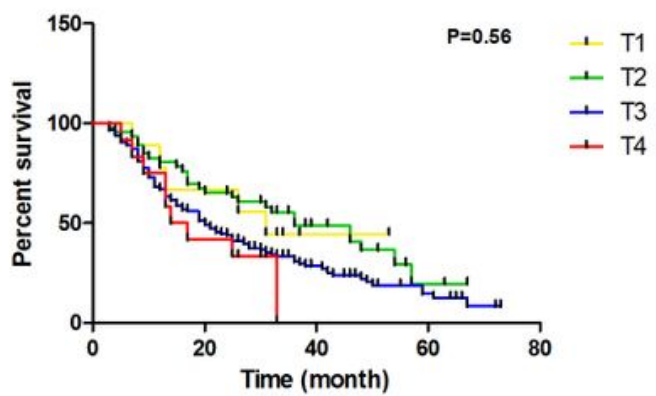

B

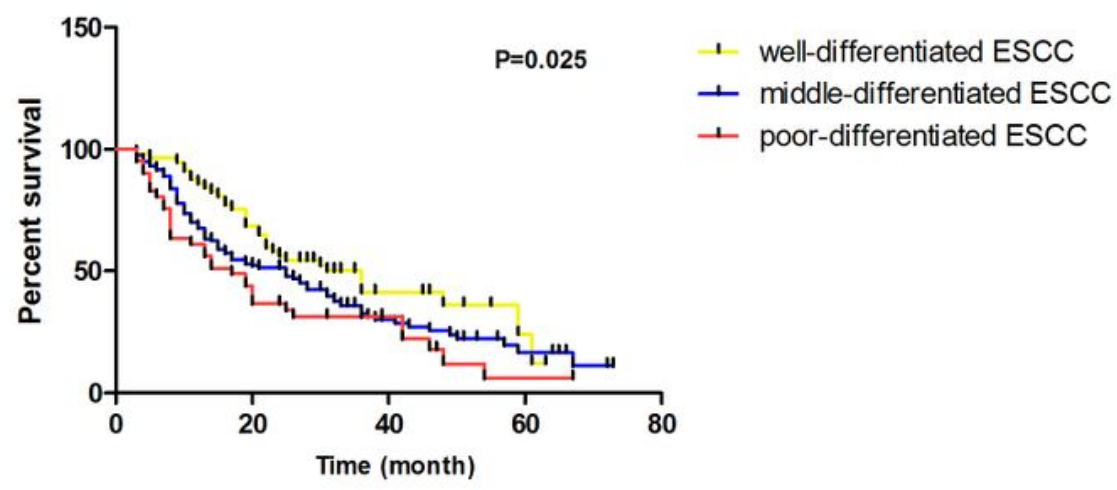

C



\section{Figure 1}

Comparison of overall survival between patients according to different $\mathrm{T}$ stages, tumor differentiation and $\mathrm{N}$ stages. Fig. 1A: The difference among sub groups which were patients with tumor $\mathrm{T}$ stage $\mathrm{T} 1, \mathrm{~T} 2$, T3, and T4. Fig. 1B: The difference among sub groups which were patients with well-differentiated ESCC, middle-differentiated ESCC and poor-differentiated ESCC. Fig. 1C: The difference among sub groups which were patients with tumor N stages N0, N1, N2 and N3. 
A.

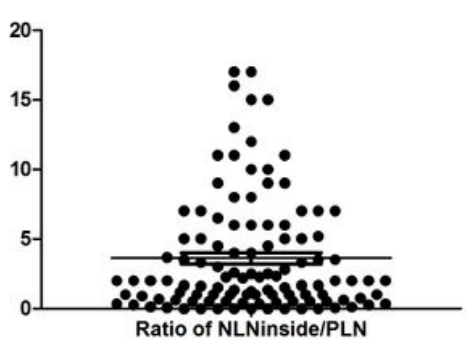

D.

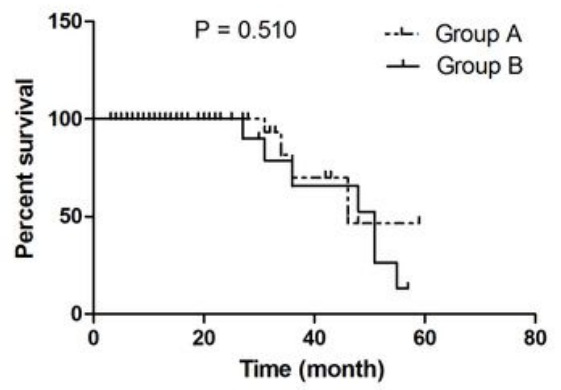

B.

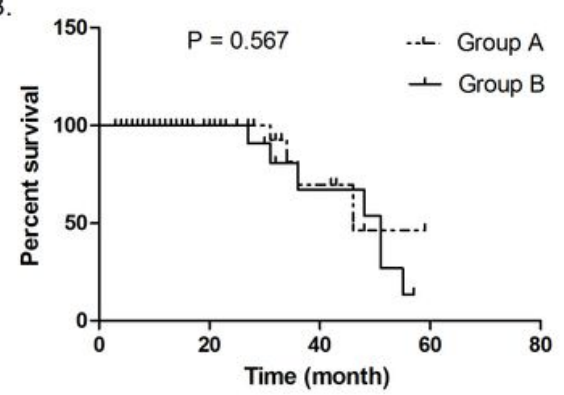

E.

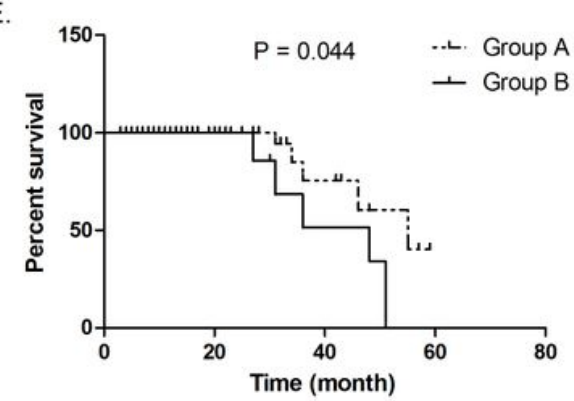

C.



F.

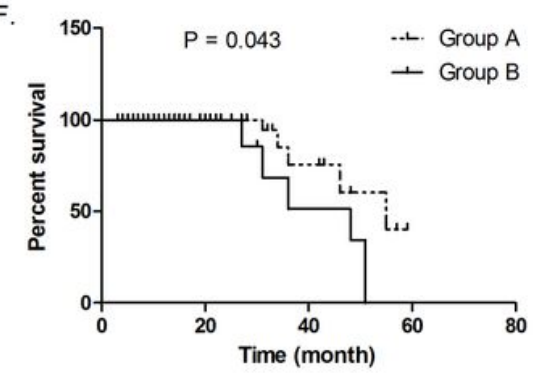

Figure 2

The investigation on the cut-off number of PLN which classified patients into two groups with different outcome Fig. 2A: The survival analysis on all the patients. Fig 2B: The group of patient with 1 PLN removed via lymphadenectomy had a better prognosis than and the other group with more than 1 PLN. Fig. $2 \mathrm{C}$ to Fig. 2N: Significant difference of prognosis were confirmed between the two groups which were classified using a cut-off number of PLN from 2 to 17. 